\title{
Análise da aplicação das métricas temporais e máximas na avaliação da poluição sonora gerada por um heliporto
}

\author{
Analysis of the application of temporal and maximum \\ metrics in the evaluation of sound pollution generated by \\ a helipad
}

\section{Luiz Antonio Perrone Ferreira de Brito Rafael Felipe Guatura João Pedro Reis da Silva}

\section{Resumo}

A poluição sonora permeia o meio urbano de diversas formas, entre elas o ruído aeronáutico. O ruído gerado por aeronaves tem um potencial de incômodo à população em geral. A normalização nacional e internacional utiliza métricas temporais, obtidas pela média das medidas instantâneas em dado período. Entretanto, mesmo em locais onde os critérios são atendidos, há um potencial de incômodo como relatado na literatura, principalmente quando da passagem de uma aeronave. Esta pesquisa teve por objetivo avaliar a aplicação das métricas temporais e máximas na quantificação da poluição sonora gerada por um heliporto. Foram executadas $70 \mathrm{~h}$ de medição de nível de pressão sonora, segundo a NBR 13368 (ABNT, 1995), e utilizadas curvas dose-resposta para avaliação dos resultados obtidos. Os resultados indicam que, ao se utilizarem as métricas temporais, os critérios normativos de avaliação são atendidos, mesmo existindo um potencial de incômodo de $30 \%$ da população. Durante as medições foram observadas diferenças de cerca de $30 \mathrm{~dB}$ entre o nível de ruído de fundo e o nível de pressão sonora máximo, gerado durante a passagem de aeronaves com uma frequência média de $12 \mathrm{~min}$. Pode-se concluir que o atendimento aos critérios de avaliação, baseados em métricas temporais, não garante à população condições de conforto acústico.

Palavras-chave: Poluição sonora. Ruído de aeronaves. Meio ambiente.
Abstract
Noise pollution in cities derives from various sources, including aeronautical noise. Aircraft noise is a potential cause of discomfort for the general population. National and international standards use temporal metrics, obtained through the logarithmic mean of instantaneous measurements over a period. But there is still potential for discomfort even in places where those criteria are met, as reported in the literature, especially when an aircraft flies over the receiver. This researchstudy aimed to evaluate the application of temporal and maximum metrics in the quantification of sound pollution generated by a helipad. Seventy hours of measurement were performed following to the procedures of NBR 13368 (ABNT, 1995) and dose-response curves were used to evaluate the results obtained. The results indicate that when using temporal metrics, the normative evaluation criteria are met, even though there is potential for discomfort of $30 \%$ of the population. During the measurements, differences of about $30 \mathrm{~dB}$ between background noise and peak noise were observed during aircraft overfly with an average frequency of $12 \mathrm{~min}$. The conclusion is that meeting the evaluation criteria based on time metrics does not guarantee the acoustic comfort of the population.
Keywords: Noise pollution. Aircraft noise. Environment.

${ }^{1}$ Luiz Antonio Perrone Ferreira de Brito 1Universidade de Taubaté Taubaté - SP - Brasil

${ }^{2}$ Rafael Felipe Guatura ¿Universidade de Taubaté Taubaté - SP - Brasil

${ }^{3}$ João Pedro Reis da Silva 3Universidade de Taubaté Taubaté - SP - Brasil

Recebido em 16/08/19 Aceito em 27/12/19 


\section{Introdução}

A Organização Mundial da Saúde (OMS) estima que a cada ano na Europa Ocidental um milhão de pessoas saudáveis tenham a saúde comprometida por algum distúrbio proveniente da poluição sonora (WORLD..., 2011). A União Europeia trata a exposição de ruído industrial, rodoviário, ferroviário e aeronáutico como um importante problema ambiental por causar efeitos adversos na qualidade de vida das pessoas e comprometer o bem-estar (LAWTON; FUJIWARA, 2016; SCHRECKENBERG et al., 2017), com efeitos na saúde, como hipertensão, estresse (LEFÈVRE; LAMBERT; LAUMON, 2017) e dificuldades de aprendizagem em crianças (MURPHY, 2014).

Segundo Marques (2015), o combate ao ruído urbano se torna cada vez mais difícil, pois está presente em todos os locais, na maioria dos casos individualmente, cada um sozinho sem apresentar desconforto significativo, porém o conjunto deles acaba por causar problemas. O planejamento urbano é essencial para a redução dos efeitos do ruído na população em geral, sendo responsável pela qualidade de vida do aglomerado urbano. As variáveis que envolvem o planejamento urbano quanto ao controle do ruído são complexas, como o tráfego de veículos e aeronaves, que está relacionado com a poluição sonora e do ar, mas, ao mesmo tempo, com efeitos benéficos à sociedade, contribuindo para a mobilidade e não devendo, portanto, simplesmente ser eliminado. O mesmo ocorre com as atividades industriais, comerciais e de entretenimento, que são importantes para a economia (BRITO, 2017).

O estudo do ruído aeronáutico, gerado pela operação de pouso, decolagem e taxiamento de aeronaves, teve início nos Estados Unidos na década de 1960, quando a comunidade exposta à poluição sonora pressionou as autoridades americanas para que houvesse um controle. A Federal Aviation Administration (FAA) publicou em 1976 a lei de política de redução de ruído de aviação intitulada Noise Abatement of 1976 (DINATO, 2011). Desde então, o número de pessoas expostas ao ruído aeronáutico nos Estados Unidos diminuiu de aproximadamente 7 milhões em 1975 para pouco mais de 400 mil em 2018. Ao mesmo tempo, o número de embarques aumentou de aproximadamente 200 milhões em 1975 para mais de 850 milhões em 2018 (FEDERAL..., 2019).

Outro fator importante na diminuição da exposição ao ruído aeronáutico nos EUA foi a cooperação entre os administradores de aeroportos, companhias aéreas, governos estaduais e locais, e comunidades para reduzir o número de pessoas que vivem em áreas próximas a aeroportos expostos a níveis significativos de ruído de aeronaves. Por meio do estudo de compatibilidade entre o uso e ocupação do solo e a poluição sonora gerada pela operação de pouso e decolagem das aeronaves foi possível impedir que receptores críticos como residências, escolas e hospitais se instalassem em locais inadequados (FEDERAL..., 2019).

A OMS orienta para que o incômodo gerado em populações próximas a aeroportos e heliportos seja monitorado e controlado, sendo indicada como metodologia a curva dose-resposta (WORLD, 2011). A curva dose-reposta permite calcular a expectativa de resposta de uma população incomodada (I\%) devido a uma fonte de ruído baseada em uma métrica temporal. Entretanto, sabe-se que o incômodo acontece justamente no momento da ocorrência do ruído indesejado, ou durante o nível de pressão sonora máximo, que não é considerado especificamente na avaliação. Nunes (2005) diagnosticou um nível de pressão sonora máximo $\left(L_{\max }\right)$ de cerca de $104 \mathrm{~dB}$ devido à passagem de aviões militares sobre escolas próximas ao Aeroporto Salgado Filho em Porto Alegre (RS, Brasil), sendo este cerca de $60 \mathrm{~dB}$ superior ao nível de ruído de fundo $\left(L_{\mathrm{rf}}\right)$. Cada vez que uma aeronave sobrevoa a escola há prejuízo na relação de ensino-aprendizagem, havendo necessidade de o assunto ser retomado quase que do início devido à perda de atenção.

Carvalho Junior (2015), em avaliação do incômodo no entorno do Aeroporto Internacional de Brasília (DF, Brasil), concluiu que $53 \%$ dos entrevistados têm o sono perturbado quando da passagem de aeronaves no período noturno, mesmo estando em uma região de nível de pressão sonora dia e noite $\left(L_{\mathrm{dn}}\right)$ de $50 \mathrm{~dB}$ a 55 dB, indicado pelo Regulamento Brasileiro da Aviação Civil 161 (RBAC 161) como critério para ocupação residencial (AGÊNCIA..., 2013). Há também o relato de interferências na atividade de leitura para $15 \%$ da população, para assistir à TV de $13 \%$, para falar ao telefone de $16 \%$ e para meditação e oração de $10 \%$ (CARVALHO JUNIOR, 2015). Evrardet al. (2017) concluíram que o ruído aeroportuário noturno está relacionado com a hipertensão masculina, porém, para que haja uma conclusão mais clara, o índice $L_{\max }$ também deveria ser considerado na análise. Assim, é possível notar que, mesmo em situações em que os critérios de nível de pressão sonora baseados em métricas temporais são atendidos, o incômodo ocorre durante $L_{\max }$.

No Brasil a NBR 13368 - Ruído gerado por aeronaves, Monitoração (ABNT, 1995), ratificada em 9 de outubro de 2017, é a norma específica para avaliação do incômodo gerado pela operação de pouso e

532 Brito, L. A. P. F. de; Guatura, R. F.; Silva, J. P. R. da 
decolagem de aeronaves. A NBR 10151 (ABNT, 2019), generalista, não é específica para o caso em questão e também adota métricas temporais. As curvas dose-resposta adotam métricas temporais em seus procedimentos de análise, assim como as normas citadas.

Em todo o Brasil existem 2.040 aeródromos e 1.180 heliportos particulares, não sendo considerado nesse universo os públicos e militares. Somente nas cidades de São Paulo e Rio de Janeiro são 245 heliportos particulares (AGÊNCIA..., 2019). Em geral, os heliportos estão localizados no topo dos edifícios das grandes cidades, atendendo a hospitais e corporações, em atividades que necessitam de agilidade e rapidez no transporte (PEREIRA, 2018).

As aeronaves de asas rotativas possuem caraterísticas peculiares de operação que as distinguem das de asa fixa, como voo a baixa altitude, indução de vibração em janelas e período de permanência em sobrevoo (FEDERAL..., 2004). As aeronaves de asas rotativas possuem frequências dominantes que se concentram entre $0,25 \mathrm{kHz}$ e $1 \mathrm{kHz}$. Segundo Vascik e Hansman (2018), as frequências predominantes de uma fonte de ruído no meio urbano que não estejam concentradas entre as oitavas de $2 \mathrm{kHz}$ e $5 \mathrm{kHz}$ têm a tendência de ser mais notadas. Segundo o relatório da Federal Aviation Administration (FEDERAL..., 2004) sobre o ruído gerado por helicópteros não militares, a percepção de um ruído também é função de quanto ele se destaca da paisagem sonora, de forma que o ruído peculiar das aeronaves de asas rotativas seria mais facilmente notado. Essa informação pode ser sustentada com os resultados obtidos por Barbosa (2011), em avaliação do incômodo gerado no entorno da Base Aérea de Santa Maria (RS, Brasil). Sua conclusão foi que o ruído gerado por helicópteros equivale ao de jatos militares, sendo mais notado que o gerado pela aviação civil.

O objetivo deste artigo foi avaliar a aplicação das métricas temporais e máximas para se obter o potencial de incômodo devido à poluição sonora gerada por um heliporto no meio urbano. Os heliportos têm o potencial de estarem mais próximos aos receptores críticos do que os aeródromos destinados a aeronaves de asas fixas, e o ruído gerado por helicópteros se destaca da paisagem sonora com mais facilidade. Essas peculiaridades tornam mais crítica a influência do $L_{\max }$ que não é incorporado de forma direta nas curvas dose-resposta existentes, nem nos critérios das normas atuais.

\section{Referencial teórico}

No Brasil os aeroportos e aeródromos civis são obrigados a ter um plano de zoneamento de ruído (PZR), podendo ser o plano básico de zoneamento de ruído (PBZR), quando houver menos de 7 mil movimentos ao ano, ou o plano específico de zoneamento de ruído (PEZR), quando o número de movimentos for superior a 7 mil por ano segundo o Regulamento Brasileiro da Aviação Civil 161 (RBAC 161) (AGÊNCIA..., 2013). O PZR representa geograficamente a área afetada pela poluição sonora gerada pelas aeronaves por meio de limitação da ocupação do solo dentro do cone de aproximação da pista de pouso que auxilia no planejamento urbano.

A métrica utilizada no PZR é o nível de pressão sonora dia e noite $\left(L_{\mathrm{dn}}\right)$, composto por outros dois índices, o nível de pressão sonora dia $\left(L_{\mathrm{d}}\right)$, medido entre $7 \mathrm{~h} 00$ e $22 \mathrm{~h} 00$, e o nível de pressão sonora noite $\left(L_{\mathrm{n}}\right)$, medido entre $22 \mathrm{~h} 00$ e $7 \mathrm{~h} 00$, este acrescido de $10 \mathrm{~dB}$ (BISTAFA, 2011). O PBZR estabelece dois tipos de classificação de áreas, uma com $L_{\mathrm{dn}}$ de $65 \mathrm{~dB}$ e outra com $75 \mathrm{~dB}$, com diferentes tipologias de ocupação do solo. O PEZR estabelece cinco áreas de classificação, com $L_{\mathrm{dn}}$ de $65 \mathrm{~dB}, 70 \mathrm{~dB}, 75 \mathrm{~dB}, 80 \mathrm{~dB}$ e $85 \mathrm{~dB}$, também com diferentes tipologias de ocupação.

O RBAC 161 (AGÊNCIA..., 2013) estabelece a necessidade de monitoramento da poluição sonora quando a média anual de movimentos de aeronaves nos últimos três anos for superior a 120 mil e possua regiões de uso residencial ou misto em mais de 50\% das áreas definidas pelas curvas de ruído $65-75 \mathrm{~dB}, 75-80 \mathrm{~dB}, 80$ $85 \mathrm{~dB}$ e acima de $85 \mathrm{~dB}$ de seu PEZR, isoladas ou conjuntamente. A metodologia e métricas desse plano não são definidas e devem ser apresentadas pelo gestor para aprovação (AGÊNCIA..., 2013).

De acordo com Carvalho Junior (2015), o RBAC 161 é adequado para o zoneamento sonoro de aeroportos, mas não é indicado para tratar do incômodo a comunidades, de modo que estar dentro de uma zona de $L_{\mathrm{dn}}$ de $65 \mathrm{~dB}$ não significa que não haverá desconforto. Assim, o principal instrumento do poder público municipal para o ordenamento urbano visando impedir a presença de população em áreas não permitidas pelo PZR não é eficiente, pois incorpora na análise apenas as métricas temporais e nem sempre é respeitado, como o ocorrido no entorno do Aeroporto Internacional de Guarulhos, SP, Brasil(ROSOLEM; HENKES, 2017). Outro ponto a se considerar é a falta de atualização do PZR devido à elevação do número de movimentos de aeronaves, o que altera a tipologia de classificação das áreas de entorno (ALENCAR; OITICICA, 2017). 
Essa situação também pode ser extrapolada para os heliportos, principalmente nas grandes cidades, onde o adensamento urbano é uma realidade (BRITO, 2017).

A NBR 13368 (ABNT, 1995), referência normativa no Brasil para a avaliação do incômodo devido às operações de pouso e decolagem de aeronaves de asas fixas e rotativas, adota como critério de avaliação a diferença dos resultados obtidos em uma medição de no mínimo 60 min entre o nível de ruído aeronáutico $\left(L_{\mathrm{ra}}\right)$ e o nível de ruído de fundo $\left(L_{\mathrm{rf}}\right)$. Entende-se nesse caso que o $L_{\mathrm{rf}}$ é o nível de ruído obtido devido a todas as fontes sonoras presentes no local de medição. O $L_{\mathrm{ra}}$ é o nível de ruído obtido quando das operações de pouso, decolagem e taxiamento das aeronaves no aeródromo. Caso a diferença entre $L_{\mathrm{ra}}$ e $L_{\mathrm{rf}}$ seja maior ou igual a $3 \mathrm{~dB}$, o impacto sonoro será considerado significativo, e quando for menor que $3 \mathrm{~dB}$, será desprezível. A NBR 13368 (ABNT, 1995) também estabelece um critério de avaliação do incômodo, como descrito na Tabela 1, sem fazer distinção do tipo de ocupação do solo, como áreas residenciais e mistas, para os parâmetros estabelecidos.

A Resolução $n^{\circ} 1$ do Conselho Nacional do Meio Ambiente (Conama), de 8 de março de 1990, indica a NBR 10151- Medição e avaliação de níveis de pressão sonora em áreas habitadas - Aplicação de uso geral como parâmetro de análise de ruído ambiental. Essa norma da ABNT estabelece o limite do nível de pressão sonora ( $\left.\mathrm{R} L_{\mathrm{Aeq}}\right)$, Tabela 2, para os períodos diurno e noturno conforme o tipo de ocupação do solo. A NBR 10151 (ABNT, 2019) é uma norma generalista para a avaliação do ruído ambiental, não específica para a análise do ruído aeronáutico, como a NBR 13368 (ABNT, 1995). As nomenclaturas das métricas também são diferentes: o $L_{\mathrm{ra}}$ e o $L_{\mathrm{rf}}$ da NBR 13368 (ABNT, 1995) equivalem ao $L_{\mathrm{Aeq}}$ e ao $L_{\mathrm{res}}$ da NBR 10151 (ABNT, 2019) respectivamente. Ambas as normas adotam métricas baseadas na média temporal de energia.

As curvas dose-resposta relacionam a expectativa de resposta de uma população incomodada (I\%) com determinada fonte de ruído (dose), podendo também ser relacionadas à saúde pública e à gestão de longo prazo. A primeira tentativa desse tipo de avaliação em aeródromos foi feita pelo modelo matemático apresentado por Schultz (1978) (Equação 1). A Federal Interagency Committee on Noise (Ficon), dos Estados Unidos, revisou o modelo matemático de Schultz com a atualização de dados de incômodo devido às mudanças ocorridas no setor aeronáutico no período, resultando na Equação 2 (FEDERAL..., 1992). A European Comission (EC), da Comunidade Europeia, também propôs um modelo matemático para o ruído aeronáutico, conforme a Equação 3 (EUROPEAN..., 2002). No Brasil, Carvalho Junior (2015) desenvolveu uma curva de dose-resposta por meio de uma pesquisa com a população lindeira ao Aeroporto Internacional de Brasília, DF, Brasil, obtendo o modelo matemático apresentado pela Equação 4.

$$
\begin{array}{lr}
\mathrm{I} \%=0,8533 L_{\mathrm{dn}}-0,041 L_{\mathrm{dn}}{ }^{2}+0,00047 L_{\mathrm{dn}}{ }^{3} & \text { Eq. } 1 \\
\mathrm{I} \%=100 /\left(1+\mathrm{e}^{\left(11,13-0,141 L_{\mathrm{dn}}\right)}\right) & \text { Eq. } 2 \\
I \%=1,460 \times 10^{-5}\left(L_{\mathrm{dn}}-37\right)^{3}+1,511 \times 10^{-2}\left(L_{\mathrm{dn}}-37\right)^{2}+1,346\left(L_{\mathrm{dn}}-37\right) & \text { Eq. } 3 \\
I \%=100 /\left(1+e^{\left(6,617+0,105 L_{\mathrm{dn}}\right)}\right) & \text { Eq. } 4
\end{array}
$$

Tabela 1 - Critério de avaliação do incômodo por operações aeroportuárias

\begin{tabular}{c|c|c}
\hline Tipos de áreas & $\begin{array}{c}\text { Diurno } \\
\mathbf{d B}\end{array}$ & $\begin{array}{c}\text { Noturno } \\
\mathbf{d B}\end{array}$ \\
\hline Sem reações ou queixas esporádicas & $L_{\mathrm{ra}}<65$ & $L_{\mathrm{ra}}<55$ \\
Queixas generalizadas - Possíveis ações da comunidade & $75>L_{\mathrm{ra}}>65$ & $65>L_{\mathrm{ra}}>55$ \\
Ações comunitárias vigorosas & $L_{\mathrm{ra}}>75$ & $L_{\mathrm{ra}}>65$ \\
\hline
\end{tabular}

Fonte: ABNT (1995).

Tabela 2 - Limite de nível de pressão sonora RLAeq

\begin{tabular}{c|c|c}
\hline Tipos de áreas & $\begin{array}{c}\text { RLAeqdiurno } \\
\text { dB }\end{array}$ & $\begin{array}{c}\mathbf{R} L_{\text {Aeqnoturno }} \\
\mathbf{d B}\end{array}$ \\
\hline Áreas de sítios e fazendas & 40 & 35 \\
Área estritamente residencial/urbana ou de escolas & 50 & 45 \\
Área mista, predomínio residencial & 55 & 50 \\
Área mista com vocação comercial e administrativa & 60 & 55 \\
Área mista com vocação recreacional & 65 & 55 \\
Área predominantemente industrial & 70 & 60 \\
\hline
\end{tabular}

Fonte: ABNT (2019). 
Nas Equações de 1 a 4, I\% é a expectativa de resposta de uma população incomodada, e $L_{\mathrm{dn}}$, o nível de pressão sonora dia e noite.

A Figura 1 mostra as curvas dose $\left(L_{\mathrm{dn}}\right)$ e resposta (I\%) propostas por Schultz (1978), Ficon (FEDERAL..., 1992), EC (EUROPEAN..., 2002) e Carvalho Junior (2015). Observa-se que as curvas mais antigas, Shultz (1978) e Ficon (FEDERAL..., 1992), apresentam diferença significativa, com menores valores de incômodo em relação às mais novas, EC (EUROPEAN..., 2002) e Carvalho Junior (2015), aparentemente uma incoerência, pois as aeronaves antigas, em geral, eram mais ruidosas, o que provocaria maior incômodo. No entanto, a população atual é mais instruída quanto a sua saúde e a seus direitos, sendo mais crítica em relação à poluição sonora (FEDERAL..., 2004).

As métricas $L_{\mathrm{ra}}, L_{\mathrm{rf}}, L_{\mathrm{Aeq}}$ e $L_{\mathrm{dn}}$ são obtidas em um período, sendo calculadas a partir da média temporal de energia das medidas instantâneas $\left(L_{\mathrm{i}}\right)$, em geral obtidas a cada segundo. A métrica $L_{\max }$ representa o máximo $L_{\mathrm{i}}$ em dado período de análise, no caso do ruído aeronáutico, no momento da passagem da aeronave sobre o receptor em questão. Na literatura é possível encontrar pesquisas que relacionam doenças, incômodo e dificuldade de aprendizagem ao $L_{\max }$. Bergtrön et al. (2015) estudaram o efeito do ruído gerado pela operação do Aeroporto de Frankfurt e evidenciaram que, mesmo com $L_{\mathrm{ra}}$ de $61 \mathrm{~dB}$, os professores relataram dificuldade de concentração das crianças, na leitura e no aprendizado. Bistafa e Bradley (2000) concluíram que $10 \mathrm{~dB}$ acima do $L_{\mathrm{rf}}$ são suficientes para atrapalhar a comunicação em uma conversa, o que corrobora a incorporação do $L_{\max }$ nos critérios de avaliação de incômodo à população.

\section{Método}

Para atender ao objetivo proposto, realizaram-se medições do nível de pressão sonora em um heliporto para determinação do $L_{\mathrm{ra}}$ e do $L_{\mathrm{rf}}$. A partir dos resultados obtidos, foi verificado se os critérios da NBR 13368 (ABNT, 1995) e NBR 10151 (ABNT, 2019) foram atendidos. Os dados obtidos também possibilitaram o cálculo do $L_{\mathrm{dn}}$ para que fosse determinada a I\% por meio das curvas dose-resposta. Os dados de I\% foram relacionados posteriormente com os critérios das normas NBR 13368 (ABNT, 1995) e NBR 10151 (ABNT, 2019), que utilizam métricas temporais. Os dados obtidos também possibilitaram quantificar o número de ocorrências do $L_{\max }$ para que fosse possível relacioná-lo a I\% e verificar sua influência no incômodo da população.

Os critérios, parâmetros de avaliação e nomenclatura das métricas da NBR 13368 (ABNT, 1995) foram utilizados para a realização das medições. A NBR 13368 (ABNT, 1995) adota como critério de avaliação a diferença dos resultados obtidos em uma medição de 60 min entre o nível de ruído aeronáutico $\left(L_{\mathrm{ra}}\right)$ e o nível de ruído de fundo $\left(L_{\mathrm{rf}}\right)$. Caso haja diferença maior ou igual a $3 \mathrm{~dB}$, o impacto sonoro será considerado significativo, e menor que $3 \mathrm{~dB}$, desprezível. Outro parâmetro de análise é o $L_{\mathrm{ra}}$, como descrito na Tabela 1 . Utilizaram-se também os critérios da NBR 10151 (ABNT, 2019), pois trata da avaliação do ruído ambiental em geral e utiliza métricas temporais. A NBR 10151 (ABNT, 2019) adota como critério de avaliação a subtração do $L_{\text {res }}\left(L_{\mathrm{rf}}\right)$ do $L_{\text {Aeq }}\left(L_{\mathrm{ra}}\right)$ para o cálculo do nível de pressão sonora específico $\left(L_{\mathrm{esp}}\right)$. O $L_{\mathrm{esp}}$ é comparado com o $\mathrm{R} L_{\text {Aeq, }}$, dado pela Tabela 2.

Figura 1 - Curvas dose-resposta propostas por Shultz (1978), Ficon (FEDERAL..., 1992), EC (EUROPEAN..., 2002) e Carvalho Junior (2015)

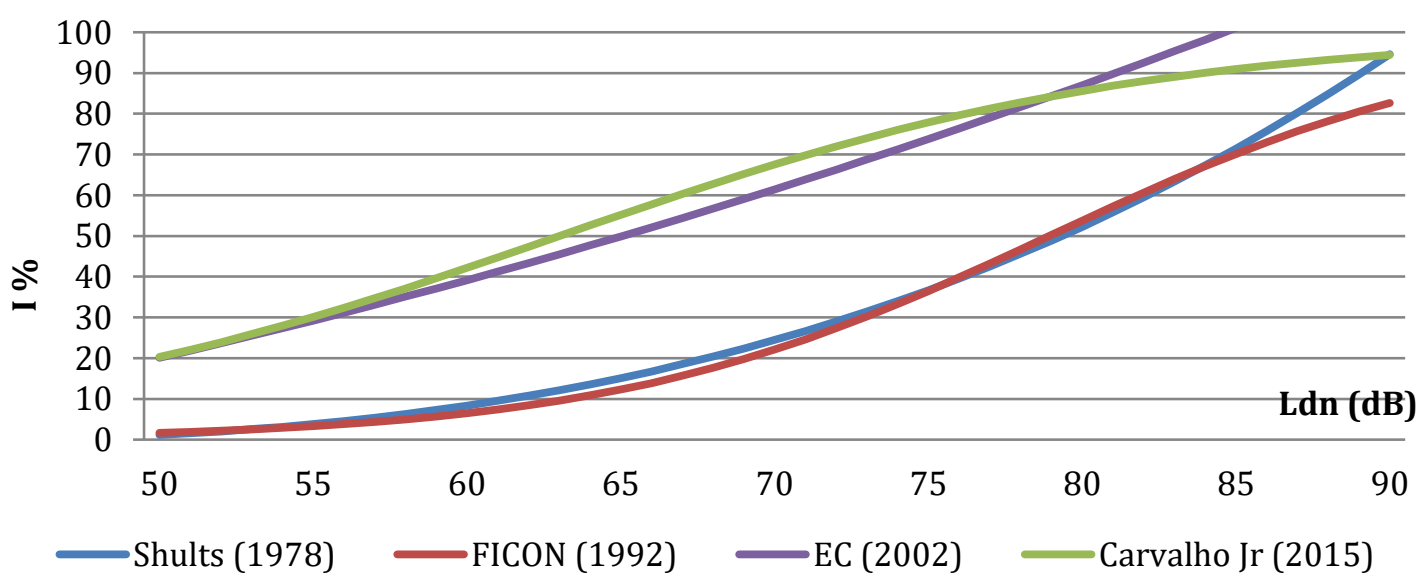


As curvas dose-resposta propostas por EC (EUROPEAN..., 2002) e Carvalho Junior (2015), Equações 3 e 4, foram utilizadas para o cálculo da porcentagem de incomodados, e este como parâmetro de avaliação, pois são as mais novas curvas dose-resposta apresentadas neste artigo, sendo a segunda obtida no Brasil, com parâmetros mais próximos da realidade atual. Na verdade, busca-se com a utilização dessas curvas verificar se a porcentagem de incomodados pode ser relacionada com as métricas temporais ou máximas. A despeito do fato de as curvas dose-resposta utilizadas terem sido elaboradas para operação de aeronaves em geral, e não especificamente para as de asas rotativas, ambas as fontes de ruído geram diferenças significativas entre as métricas temporais e máximas (NUNES, 2005; BERGTRÖN et al., 2015; VASCIK; HANSMAN, 2018). Segundo o relatório da Federal Aviation Administration (FEDERAL..., 2004) sobre o ruído gerado por helicópteros não militares, haveria necessidade de correções na I\% somente se nas avaliações internas das edificações houvesse acréscimo de $3 \mathrm{~dB}$ a $5 \mathrm{~dB}$ ao $L_{\mathrm{ra}}$, e não em ambientes externos, como conduzido nesta pesquisa. O horário de operação de heliporto avaliado é das $6 \mathrm{~h}$ às $22 \mathrm{~h}$, sem operações noturnas. Dessa forma, o $L_{\mathrm{ra}}$ obtido nas $10 \mathrm{~h}$ de medições diárias foi considerado como $L_{\mathrm{dn}}$, neste caso composto apenas do $L_{\mathrm{d}}$. Segundo Silva (2016), a métrica $L_{\mathrm{d}}$ é a que apresenta a melhor correlação entre a dose $\left(L_{\mathrm{d}}\right)$ e a resposta (I\%).

Os procedimentos de medição e calibração utilizados foram os da NBR 10151 (ABNT, 2000), pois na época da realização do levantamento de dados essa versão da norma estava em vigor. Os equipamentos foram posicionados a 1,2 $\mathrm{m}$ do solo e a mais de $3 \mathrm{~m}$ de superfícies reflexivas, como muros e paredes, com protetor de vento no tempo de resposta fast e com a curva de ponderação "A". Durante as medições não ocorreram precipitações ou outras interferências climáticas que pudessem alterar os resultados.

O sonômetro utilizado para obter o $L_{\mathrm{ra}}$ e o $L_{\mathrm{rf}}$ foi um Larson Davis LXT, série 0001990, microfone capacitivo PCB 377B02, série 109133. Esse equipamento registrou o nível de pressão sonora durante todo o período, seja o gerado pelo heliporto, seja o gerado pelas fontes de ruído urbano. Para a obtenção apenas do $L_{\mathrm{rf}}$, foi utilizado um sonômetro Quest 2200, série KOG050005, colocado no modo pausa quando o ruído gerado pelo pouso, decolagem, taxiamento ou operação de manutenção de um helicóptero se tornava perceptível. O calibrador acústico utilizado foi um Larson Davis CAL 200, série 10603. A checagem e o ajuste de calibração foram realizados no início do período de medição e depois, a cada $3 \mathrm{~h}$, devido à alteração da temperatura ambiente. No final de cada período de medição foi executada a verificação da precisão do equipamento.

As medições foram realizadas entre $8 \mathrm{~h} 00$ e $18 \mathrm{~h} 00$ por 7 dias consecutivos no mês de maio de 2018. A incerteza de medição dos resultados obtidos é de $0,2 \mathrm{~dB}$, calculada conforme indicado pela NBR 10152 (ABNT, 2017). As medições foram realizadas em uma área aberta privada, sem obstáculos, a $465 \mathrm{~m}$ do ponto de pouso das aeronaves (Figuras 2 e 3 ). A cada hora foram anotados os resultados do $L_{\mathrm{ra}}$ e $L_{\mathrm{rf}}$, métricas definidas pela NBR 13368 (ABNT, 1995) e adotadas neste trabalho.

A NBR 13368 (ABNT, 1995) não aborda o ruído aeronáutico sobre o aspecto do nível de pressão sonora máximo $\left(L_{\max }\right)$ somente no aspecto da média temporal de energia. O mesmo ocorre com a NBR 10151 (ABNT, 2019). Segundo Bistafa e Bradley (2000), a voz humana tem nível de pressão sonora de $65 \mathrm{~dB}$ em valores médios (masculina e feminina) em esforço normal a $1,5 \mathrm{~m}$ de distância. Os autores alertam que, na presença de outras fontes de ruído $5 \mathrm{~dB}$ inferiores, há o potencial de interferência na conversação de duas pessoas, como na atividade de ensino, por exemplo. Dessa forma, durante as medições foram anotadas todas as interferências que ultrapassaram os $60 \mathrm{~dB}$ para que fosse possível avaliar os efeitos do nível de pressão sonora máximo nos receptores, seja gerado pela operação do heliporto, seja gerado por fontes urbanas. Como ruído urbano se entende o gerado pelo tráfego de veículos, leves e pesados, o gerado por empresas, latidos, música e pessoas conversando na rua, e sobrevoo de aviões e helicópteros em rotas de passagem, por exemplo. Ao final de cada dia e de todo o período de medição ( 7 dias) foram calculadas as médias temporais das medições realizadas para determinação do $L_{\mathrm{ra}} \mathrm{e}$ do $L_{\mathrm{rf}}$ do período de medição.

\section{Resultados e análise}

As Tabelas 3 a 6 apresentam os resultados horários de $L_{\mathrm{ra}}$ e de $L_{\mathrm{rf}}$ obtidos nos 7 dias consecutivos de medição, a diferença (DIF) entre o $L_{\mathrm{ra}}$ e o $L_{\mathrm{rf}}$ segundo a NBR 13368 (ABNT, 1995), e o $L_{\mathrm{es}}$ pde acordo com a NBR 10151 (ABNT, 2019). A Tabela 7 apresenta a consolidação dos dados para cada dia de medição com a média logarítmica dos resultados diários. As células sombreadas indicam o horário em que os critérios adotados não foram atendidos. As células contendo hífen representam as situações com valores de $L_{\text {esp }}$ indefinidos, pois $L_{\mathrm{ra}}$ e $L_{\mathrm{rf}}$ são iguais. 
Das 70 medições realizadas (60 min cada), 51 delas atenderam ao critério da NBR 13368 (ABNT, 1995) $\left(L_{\mathrm{ra}}\right.$ $-L_{\mathrm{rf}}<3 \mathrm{~dB}$ ), e 19 não atenderam. Durante toda a avaliação, o $L_{\mathrm{ra}}$ foi inferior ao critério (NBR 13368, ABNT 1995) para o período diurno de $65 \mathrm{~dB}$, indicando não haver reações ou apenas queixas esporádicas, conforme dados das Tabelas 1 a 6 , inclusive sendo limítrofe ao critério noturno de $55 \mathrm{~dB}$. Ao se avaliarem os dados apresentados na Tabela 7, com o $L_{\mathrm{ra}}$ e o $L_{\mathrm{rf}}$ consolidados para as $10 \mathrm{~h}$ de medições diárias, dos 7 dias de avaliação, 6 atenderam ao critério da NBR 13368 (ABNT, 1995) com a diferença entre $L_{\mathrm{ra}}$ e $L_{\mathrm{rf}}$ inferior a $3 \mathrm{~dB}$; apenas o dia 6 não atendeu. $\mathrm{O} L_{\mathrm{ra}}$ nesse dia $(53,1 \mathrm{~dB})$ foi compatível com o obtido nos demais, sendo a alteração no $L_{\mathrm{rf}}(47,3 \mathrm{~dB})$ inferior aos demais dias de medição. $O$ sexto dia de medição foi um domingo. Considerando todo o período de medição $(70 \mathrm{~h})$, o $L_{\mathrm{ra}}$ foi de $53,0 \mathrm{~dB}$, e o $L_{\mathrm{rf}}$ de $50,4 \mathrm{~dB}$, obtidos pela média temporal de energia dos dados apresentados na Tabela 7, o que atende a todos os critérios da NBR 13368 (ABNT, 1995).

Figura 2 - Vista dos equipamentos de medição

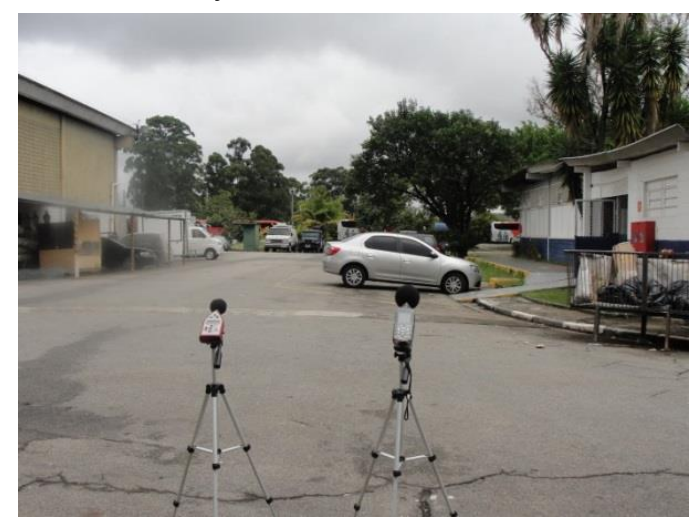

Figura 3 - Vista de um helicóptero em operação de pouso

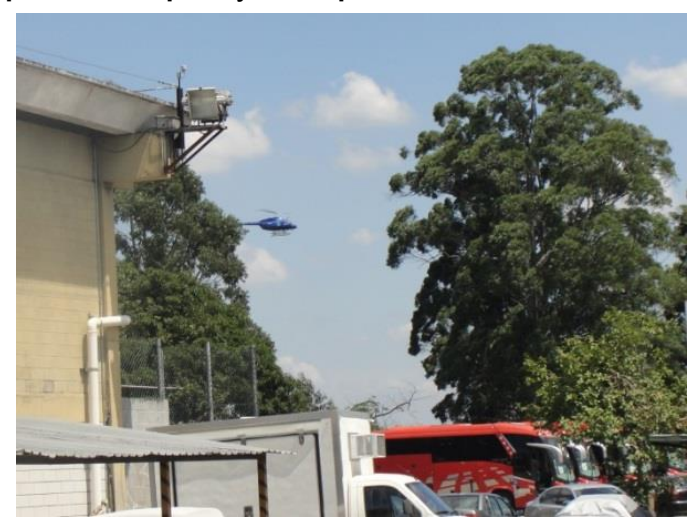

Tabela 3 - Resultados obtidos nos dias 1 e 2 para $L_{r a}$, $L_{r f}$, DIF ( $\left.L_{r a}-L_{r f}\right)$ e $L_{e s p}$

\begin{tabular}{|c|c|c|c|c|c|c|c|c|}
\hline Horário & $\begin{array}{c}L_{\mathrm{ra}} \\
\text { dB } \\
\text { Dia 1 }\end{array}$ & $\begin{array}{c}L_{\mathrm{rf}} \\
\text { dB } \\
\text { Dia 1 }\end{array}$ & $\begin{array}{c}\text { DIF } \\
\text { dB } \\
\text { Dia 1 }\end{array}$ & $\begin{array}{c}\text { Lesp } \\
\text { dB } \\
\text { Dia 1 }\end{array}$ & $\begin{array}{c}L_{\mathrm{ra}} \\
\text { dB } \\
\text { Dia } 2\end{array}$ & $\begin{array}{c}L_{\mathrm{rf}} \\
\text { dB } \\
\text { Dia } 2\end{array}$ & $\begin{array}{c}\text { DIF } \\
\text { dB } \\
\text { Dia } 2\end{array}$ & $\begin{array}{c}\text { Lesp } \\
\text { dB } \\
\text { Dia } 2\end{array}$ \\
\hline 08h00-9h00 & 53,5 & 52,4 & 1,1 & 47,0 & 54,7 & 52,3 & 2,4 & 51,0 \\
\hline 09h00-10h00 & 53,9 & 52,3 & 1,6 & 48,8 & 51,7 & 49,5 & 2,2 & 47,7 \\
\hline $10 \mathrm{~h} 00-11 \mathrm{~h} 00$ & 51,5 & 48,8 & 2,7 & 48,2 & 49,9 & 47,3 & 2,6 & 46,4 \\
\hline $11 \mathrm{~h} 00-12 \mathrm{~h} 00$ & 52,8 & 51,2 & 1,6 & 47,7 & 51,4 & 49,5 & 1,9 & 46,9 \\
\hline $12 \mathrm{~h} 00-13 \mathrm{~h} 00$ & 55,8 & 54,4 & 1,4 & 50,2 & 54,2 & 51,2 & 3,0 & 51,2 \\
\hline $13 \mathrm{~h} 00-14 \mathrm{~h} 00$ & 51,5 & 49,8 & 1,7 & 46,6 & 53,4 & 50,2 & 3,2 & 50,6 \\
\hline $14 \mathrm{~h} 00-15 \mathrm{~h} 00$ & 52,5 & 50,0 & 2,5 & 48,9 & 52,5 & 50,0 & 2,5 & 48,9 \\
\hline $15 \mathrm{~h} 00-16 \mathrm{~h} 00$ & 53,0 & 52,4 & 0,6 & 44,1 & 55,4 & 49,6 & 5,8 & 54,1 \\
\hline $16 \mathrm{~h} 00-17 \mathrm{~h} 00$ & 52,3 & 51,8 & 0,5 & 42,7 & 55,6 & 52,3 & 3,3 & 52,9 \\
\hline $17 \mathrm{~h} 00-18 \mathrm{~h} 00$ & 51,7 & 51,1 & 0,6 & 42,8 & 52,9 & 52,7 & 0,2 & 39,4 \\
\hline
\end{tabular}


Tabela 4 - Resultados obtidos nos dias 3 e 4 para $L_{r a}$, $L_{r f}$, DIF $\left(L_{r a}-L_{r f}\right)$ e $L_{e s p}$

\begin{tabular}{c|c|c|c|c|c|c|c|c}
\hline HORÁRIO & $\begin{array}{c}\boldsymbol{L}_{\mathbf{r a}} \\
\mathbf{d B} \\
\text { Dia 3 }\end{array}$ & $\begin{array}{c}\boldsymbol{L}_{\mathbf{r f}} \\
\mathbf{d B} \\
\text { Dia 3 }\end{array}$ & $\begin{array}{c}\text { DIF } \\
\mathbf{d B} \\
\text { Dia 3 }\end{array}$ & $\begin{array}{c}\boldsymbol{L}_{\text {esp }} \\
\mathbf{d B} \\
\text { Dia 3 }\end{array}$ & $\begin{array}{c}\boldsymbol{L}_{\mathbf{r a}} \\
\mathbf{d B} \\
\text { Dia 4 }\end{array}$ & $\begin{array}{c}\boldsymbol{L}_{\mathbf{r f}} \\
\mathbf{d B} \\
\text { Dia 4 }\end{array}$ & $\begin{array}{c}\text { DIF } \\
\mathbf{d B} \\
\text { Dia 4 }\end{array}$ & $\begin{array}{c}\boldsymbol{L}_{\text {esp }} \\
\mathbf{d B} \\
\text { Dia 4 }\end{array}$ \\
\hline 08h00-9h00 & 51,7 & 50,2 & 1,5 & 46,4 & 56,8 & 54,3 & 2,5 & 53,2 \\
09h00-10h00 & 54,3 & 51,4 & 2,9 & 51,2 & 56,9 & 54,2 & 2,7 & 53,6 \\
10h00-11h00 & 54,1 & 49,5 & 4,6 & 52,3 & 55,0 & 52,9 & 2,1 & 50,8 \\
11h00-12h00 & 59,2 & 58,5 & 0,7 & 50,9 & 53,1 & 50,6 & 2,5 & 49,5 \\
12h00-13h00 & 51,6 & 50,7 & 0,9 & 44,3 & 57,0 & 52,9 & 4,1 & 54,9 \\
13h00-14h00 & 55,6 & 52,9 & 2,7 & 52,3 & 54,9 & 53,5 & 1,4 & 49,3 \\
14h00-15h00 & 56,4 & 53,9 & 2,5 & 52,8 & 56,1 & 54,2 & 1,9 & 51,6 \\
15h00-16h00 & 57,1 & 53,2 & 3,9 & 54,8 & 57,0 & 54,9 & 2,1 & 52,8 \\
16h00-17h00 & 56,4 & 54,1 & 2,3 & 52,5 & 55,8 & 54,5 & 1,3 & 49,9 \\
17h00-18h00 & 55,1 & 51,2 & 3,9 & 52,8 & 54,4 & 53,6 & 0,8 & 46,7 \\
\hline
\end{tabular}

Tabela 5 - Resultados obtidos nos dias 5 e 6 para $L_{a}$, $L_{r f}$, DIF ( $\left.L_{r a}-L_{r f}\right)$ e $L_{\text {esp }}$

\begin{tabular}{c|c|c|c|c|c|c|c|c}
\hline HORÁRIO & $\begin{array}{c}\boldsymbol{L}_{\mathbf{r a}} \\
\mathbf{d B} \\
\text { Dia 5 }\end{array}$ & $\begin{array}{c}\boldsymbol{L}_{\mathbf{r f}} \\
\mathbf{d B} \\
\text { Dia 5 }\end{array}$ & $\begin{array}{c}\text { DIF } \\
\mathbf{d B} \\
\text { Dia 5 }\end{array}$ & $\begin{array}{c}\boldsymbol{L}_{\text {esp }} \\
\mathbf{d B} \\
\text { Dia 5 }\end{array}$ & $\begin{array}{c}\boldsymbol{L}_{\mathbf{r a}} \\
\mathbf{d B} \\
\text { Dia 6 }\end{array}$ & $\begin{array}{c}\boldsymbol{L}_{\mathbf{r f}} \\
\mathbf{d B} \\
\text { Dia 6 }\end{array}$ & $\begin{array}{c}\text { DIF } \\
\mathbf{d B} \\
\text { Dia 6 }\end{array}$ & $\begin{array}{c}\boldsymbol{L}_{\text {esp }} \\
\mathbf{d B} \\
\text { Dia 6 }\end{array}$ \\
\hline 08h00-9h00 & 52,7 & 48,1 & 4,6 & 50,9 & 45,5 & 44,4 & 1,1 & 39,0 \\
09h00-10h00 & 54,6 & 52,5 & 2,1 & 50,4 & 51,6 & 47,0 & 4,6 & 49,8 \\
10h00-11h00 & 50,6 & 47,4 & 3,2 & 47,8 & 53,9 & 46,4 & 7,5 & 53,0 \\
11h00-12h00 & 54,8 & 51,9 & 2,9 & 51,7 & 53,5 & 49,7 & 3,8 & 51,2 \\
12h00-13h00 & 54,2 & 52,0 & 2,2 & 50,2 & 52,3 & 46,6 & 5,7 & 50,9 \\
$13 \mathrm{~h} 00-14 \mathrm{~h} 00$ & 52,9 & 51,0 & 1,9 & 48,4 & 46,1 & 46,1 & 0 & - \\
14h00-15h00 & 54,7 & 51,9 & 2,8 & 51,5 & 50,1 & 49,1 & 1,0 & 43,2 \\
15h00-16h00 & 54,2 & 52,8 & 1,4 & 48,6 & 53,0 & 46,5 & 6,5 & 51,9 \\
16h00-17h00 & 52,0 & 48,1 & 3,9 & 49,7 & 58,6 & 51,1 & 7,5 & 57,7 \\
17h00-18h00 & 52,7 & 50,4 & 2,3 & 48,8 & 54,3 & 48,4 & 5,9 & 53,0 \\
\hline
\end{tabular}

Tabela 6 - Resultados obtidos no dia 7 para $L_{r a}, L_{r f}$, DIF ( $\left.L_{r a}-L_{r f}\right)$ e $L_{e s p}$

\begin{tabular}{c|c|c|c|c}
\hline HORÁRIO & $\begin{array}{c}\boldsymbol{L}_{\mathbf{r a}} \\
\mathbf{d B} \\
\text { Dia 7 }\end{array}$ & $\begin{array}{c}\boldsymbol{L}_{\mathbf{r f}} \\
\mathbf{d B} \\
\text { Dia 7 }\end{array}$ & $\begin{array}{c}\text { DIF } \\
\mathbf{d B} \\
\text { Dia 7 }\end{array}$ & $\begin{array}{c}\boldsymbol{L}_{\text {esp }} \\
\mathbf{d B} \\
\text { Dia 7 }\end{array}$ \\
\hline 08h00-9h00 & 56,3 & 53,8 & 2,5 & 52,7 \\
09h00-10h00 & 53,4 & 49,3 & 4,1 & 51,3 \\
10h00-11h00 & 56,4 & 54,5 & 1,9 & 51,9 \\
11h00-12h00 & 55,3 & 52,2 & 3,1 & 52,4 \\
12h00-13h00 & 55,1 & 53,9 & 1,2 & 48,9 \\
$13 \mathrm{~h} 00-14 \mathrm{~h} 00$ & 54,9 & 54,1 & 0,8 & 47,2 \\
1 14h00-15h00 & 54,8 & 54,4 & 0,4 & 44,2 \\
15h00-16h00 & 51,8 & 51,8 & 0 & - \\
16h00-17h00 & 54,5 & 52,6 & 1,9 & 50,0 \\
17h00-18h00 & 53,2 & 52,8 & 0,4 & 42,6 \\
\hline
\end{tabular}


Tabela 7 - Resultados obtidos consolidados para os 7 dias de medição para $L_{r a}, L_{r f}$, DIF ( $\left.L_{r a}-L_{r f}\right)$ e $L_{\text {esp }}$

\begin{tabular}{c|c|c|c|c}
\hline Dia & $\begin{array}{c}\boldsymbol{L}_{\mathbf{r a}} \\
\mathbf{d B} \\
\text { Consolidado }\end{array}$ & $\begin{array}{c}\boldsymbol{L}_{\mathbf{r f}} \\
\mathbf{d B} \\
\text { Consolidado }\end{array}$ & $\begin{array}{c}\text { DIF } \\
\mathbf{d B} \\
\text { Consolidado }\end{array}$ & $\begin{array}{c}\boldsymbol{L}_{\text {esp }} \\
\mathbf{d B} \\
\text { Consolidado }\end{array}$ \\
\hline 1 & 52,6 & 51,2 & 1,4 & 46,9 \\
2 & 53,2 & 50,5 & 2,7 & 49,8 \\
3 & 55,2 & 52,9 & 2,3 & 51,3 \\
4 & 55,4 & 53,2 & 2,2 & 51,4 \\
5 & 52,9 & 50,4 & 2,5 & 49,3 \\
6 & 53,1 & 47,3 & 5,8 & 51,8 \\
7 & 54,1 & 52,1 & 2,0 & 49,8 \\
\hline
\end{tabular}

Ao se avaliarem os resultados obtidos pelos critérios da NBR 10151 (ABNT, 2019) em todas as medições, o $L_{\text {esp }}$ foi inferior ao estabelecido para regiões residenciais de uso misto $\left(R L_{\mathrm{Aeq}}=55 \mathrm{~dB}\right.$ ), e 14 foram inferiores ao critério de zona residencial $\left(\mathrm{R} L_{\mathrm{Aeq}}=50 \mathrm{~dB}\right)$. Dessa forma, o $L_{\mathrm{ra}}$ está compatível com os critérios adotados para essas tipologias de ocupação urbana segundo a NBR 10151 (ABNT, 2019). Considerando o resultado consolidado para as $10 \mathrm{~h}$ de medições diárias, Tabela 7 , o $L_{\text {esp }}$ foi inferior ao critério do zoneamento residencial de uso misto em todas as situações, e em quatro delas foi inferior ao critério residencial. Ao se avaliar todo o período de medição o $L_{\mathrm{esp}}(49,4 \mathrm{~dB})$, foi inferior ao critério para zonas residenciais.

Os critérios da NBR 13368 (ABNT, 1995) e da NBR 10151 (ABNT, 2019) foram praticamente atendidos, o que, em princípio, implicaria menores expectativas de incômodo devido à operação das aeronaves.

A Tabela 8 apresenta a expectativa de resposta de uma população incomodada (I\%), obtida a partir das curvas dose-resposta propostas por EC (2002) e Carvalho Junior (2015). Os valores de I\% variaram entre $25 \%$ e $30 \%$, que seria a parte de uma população que teoricamente se incomodaria com a operação do heliporto. Esse resultado, de certa forma, se contrapõe à avaliação obtida com base na NBR 13368 (ABNT, 1995), ou seja, sem reações ou queixas esporádicas $\left(L_{\mathrm{ra}}<65 \mathrm{~dB}\right.$ - período diurno) e aos critérios da NBR 10151 (ABNT, 2019). Ora, se há o potencial de 30\% da população estar incomodada, não seria razoável esperar apenas queixas esporádicas e o atendimento das normas vigentes.

Dessa forma, pode-se supor que a expectativa de resposta de uma população incomodada de cerca de $30 \%$ seja oriunda não do nível de pressão sonora equivalente, média temporal de energia sonora, nesta pesquisa tratado como $L_{\mathrm{ra}}$, como indica a NBR 13368 (ABNT, 1995), mas do nível de pressão sonora máximo $\left(L_{\max }\right)$.

Santos (2015) avaliou o incômodo das operações de pouso e decolagem do Aeroporto Internacional de Guarulhos, SP, Brasil, e concluiu que um dos fatores que melhoraram a precisão das curvas dose-resposta foi a inclusão da sensação do aumento e da redução do ruído aeronáutico, considerando-se o $L_{\max } \operatorname{nos}$ algoritmos de cálculo. Da mesma forma, Silva (2016) concluiu a necessidade da inclusão do $L_{\max }$ nas métricas de avaliação da I\%. As Figuras 4 a 10 ilustram o histórico do tempo das medidas instantâneas $\left(L_{\mathrm{i}}\right)$, a evolução do $L_{\mathrm{ra}}$, do $L_{\mathrm{rf}}$ e do $L_{\mathrm{rf}}$ acrescido de $3 \mathrm{~dB}$ para os 7 dias de medições, com $10 \mathrm{~h}$ de avaliação diárias.

É possível observar que a diferença de valores entre o $L_{\mathrm{ra}}$, linha vermelha, e o $L_{\max }$, representado pelo pico de valores das linhas azuis, é significativa. Observa-se também que a quantidade de ocorrências do nível de pressão sonora máximo no período de análise também é elevada. A Tabela 9 apresenta as ocorrências $\left(L_{\max }>\right.$ $60 \mathrm{~dB}$ ) anotadas durante o período de avaliação e o $L_{\max }$ relacionado à operação do heliporto e a outras fontes de ruído urbano. No total foram 300 (43,1\%) ocorrências relacionadas à operação do heliporto e 396 $(56,9 \%)$ relacionadas ao ruído urbano. O maior valor de $L_{\max }$ relacionado ao heliporto foi de $79,5 \mathrm{~dB}$, obtido a $465 \mathrm{~m}$ do local de pouso, e o relacionado às fontes de ruído urbano foi de 73,9 dB. Como parâmetro de comparação, o nível de pressão sonora estimado para a Avenida Paulista é de $79 \mathrm{~dB}$, e para a Times Square, em New York, EUA, de 80 dB (SHOEGIMA, 2011). 
Tabela 8 - Porcentagem de expectativa de incômodo (I\%) a partir das equações propostas por EC (EUROPEAN..., 2002) e Carvalho Junior (2015)

\begin{tabular}{c|c|c|c}
\hline Dia & $\begin{array}{c}\boldsymbol{L}_{\mathbf{d n}} \\
\text { dB }\end{array}$ & $\begin{array}{c}\text { I\% } \\
\text { Carvalho Junior (2015) }\end{array}$ & $\begin{array}{c}\text { I\% } \\
\text { EC (2002) }\end{array}$ \\
\hline 1 & 52,7 & 25 & 25 \\
2 & 53,7 & 27 & 27 \\
3 & 55,6 & 32 & 30 \\
4 & 55,6 & 32 & 30 \\
5 & 53,6 & 27 & 27 \\
6 & 53,3 & 37 & 26 \\
7 & 54,5 & 29 & 28 \\
\hline
\end{tabular}

Figura 4 - Histórico do tempo das medidas instantâneas $\left(L_{i}\right)$, a evolução do $L_{r a}$, do $L_{r f}$ e do $L_{r f}$ acrescido de $3 \mathrm{~dB}$ (ABNT 1995), dia 1

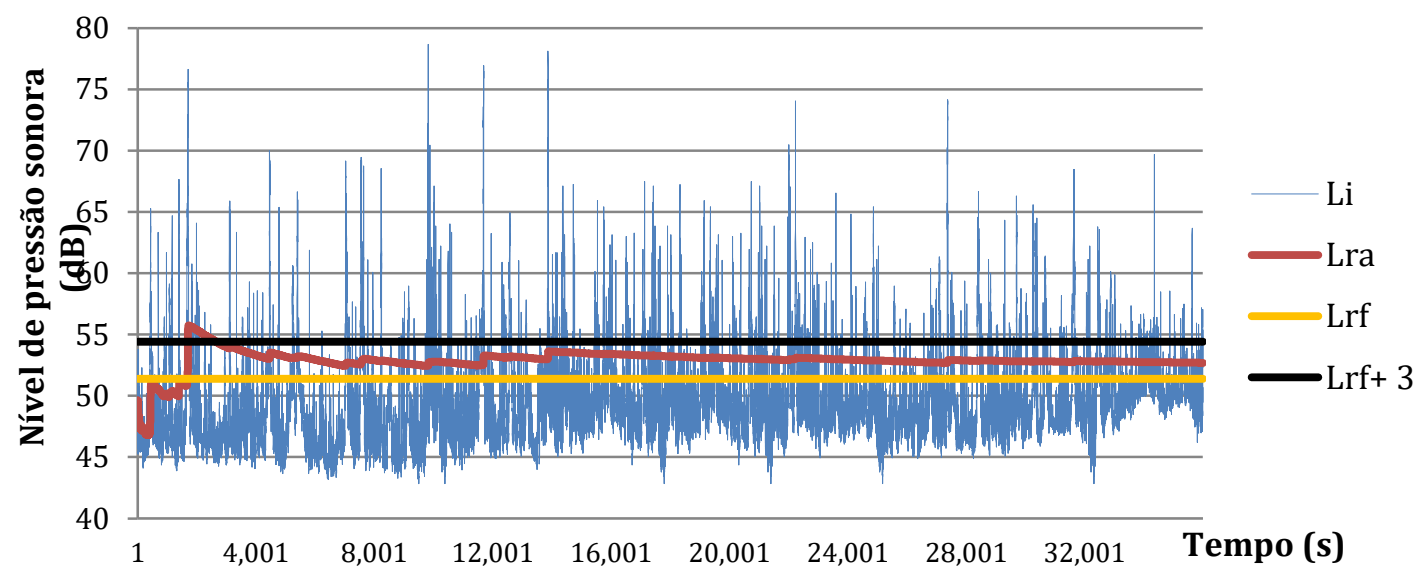

Figura 5 - Histórico do tempo das medidas instantâneas $\left(L_{i}\right)$, a evolução do $L_{r a}$, do $L_{r f}$ e do $L_{r f}$ acrescido de $3 \mathrm{~dB}$ (ABNT 1995), dia 2

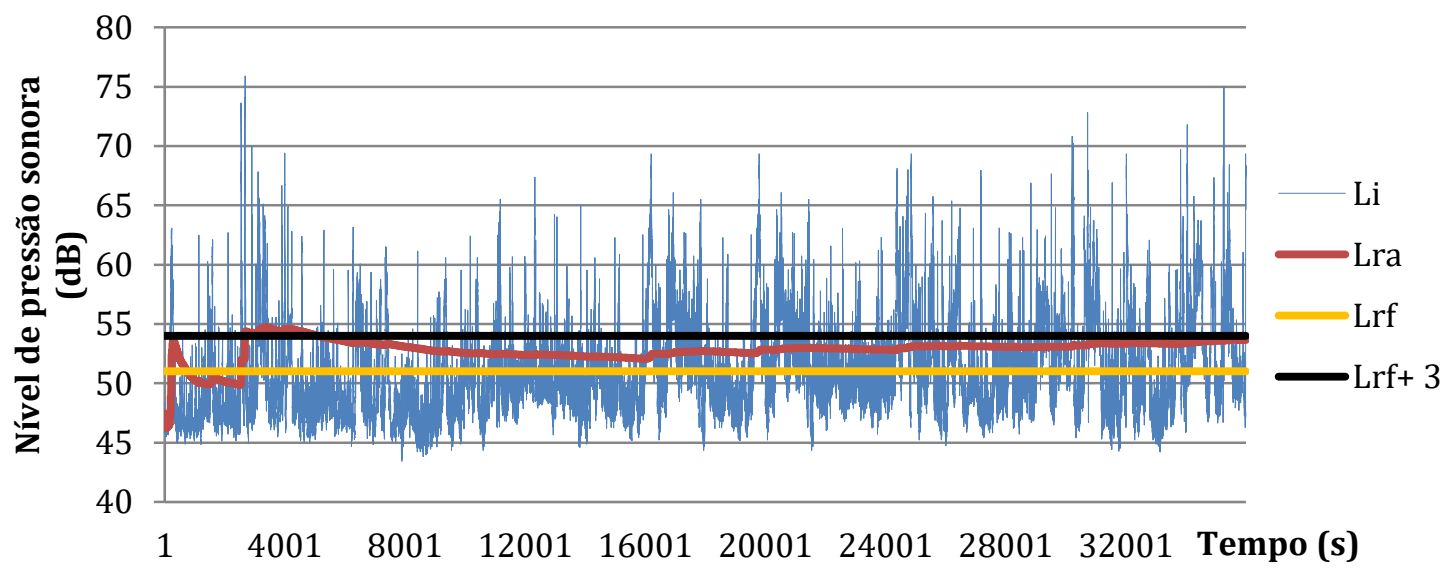


Figura 6 - Histórico do tempo das medidas instantâneas $\left(L_{i}\right)$, a evolução do $L_{r a}$, do $L_{r f}$ e do $L_{r f}$ acrescido de 3 dB (ABNT 1995), dia 3

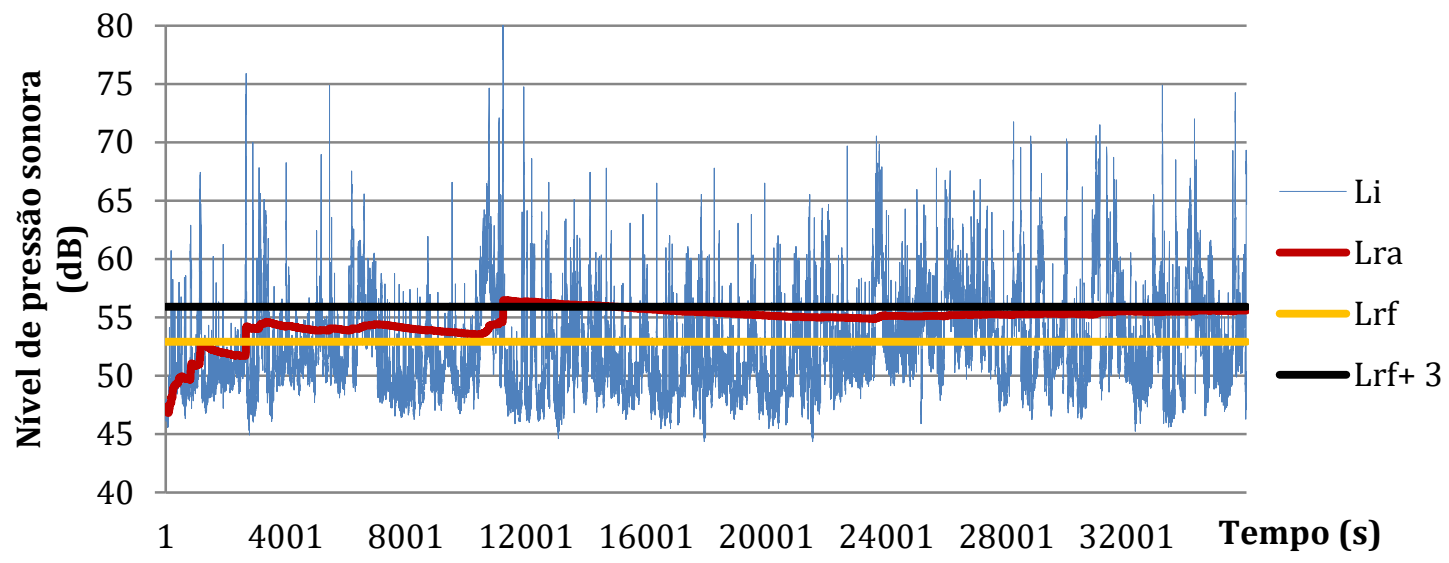

Figura 7 - Histórico do tempo das medidas instantâneas $\left(L_{i}\right)$, a evolução do $L_{r a}$, do $L_{r f}$ e do $L_{r f}$ acrescido de 3 dB (ABNT 1995), dia 4

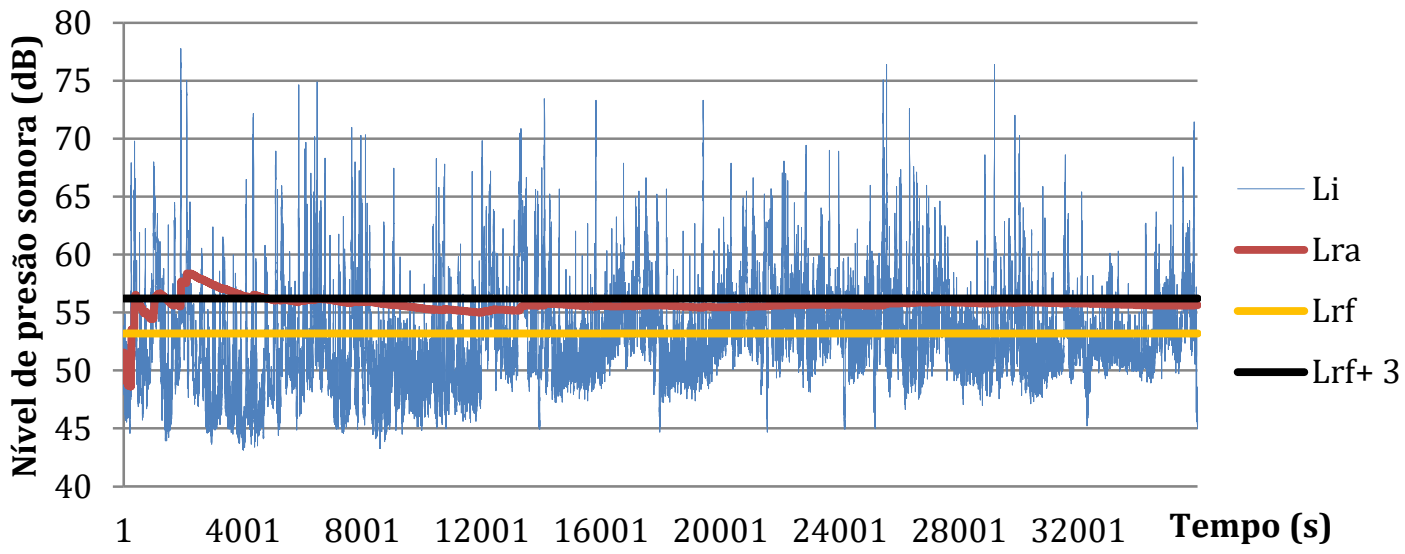

Figura 8 - Histórico do tempo das medidas instantâneas $\left(L_{i}\right)$, a evolução do $L_{r a}$, do $L_{r f e}$ do $L_{r f}$ acrescido de 3 dB (ABNT 1995), dia 5

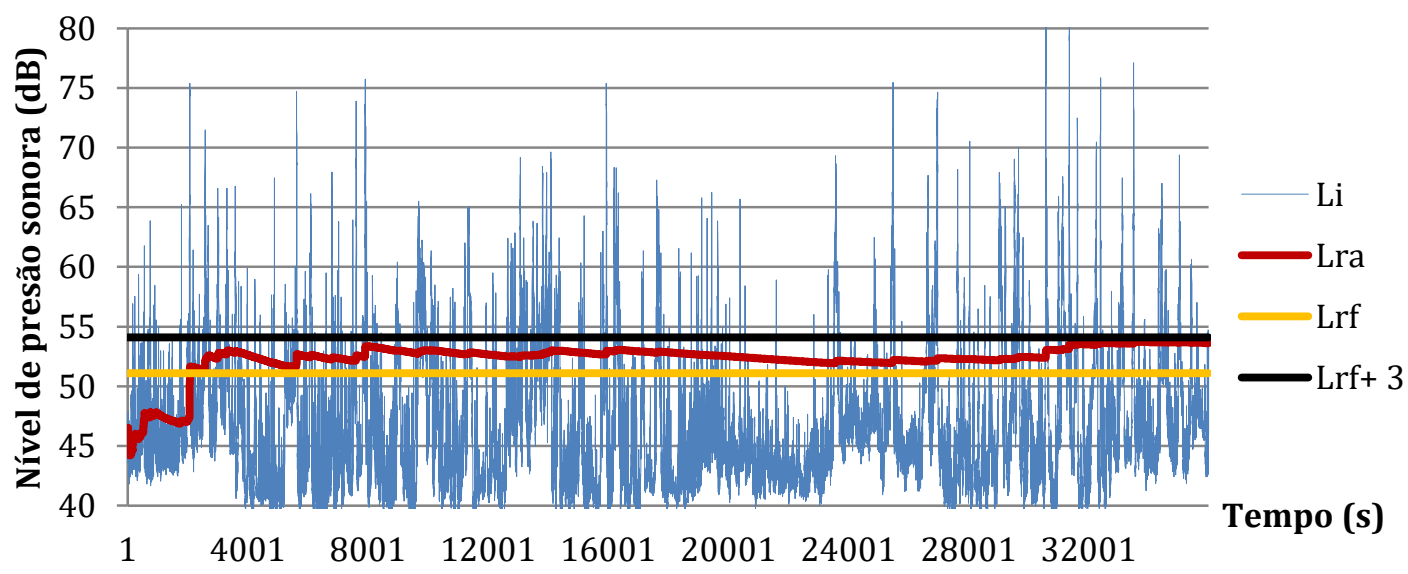


Figura 9 - Histórico do tempo das medidas instantâneas $\left(L_{\mathrm{i}}\right)$, a evolução do $L_{\mathrm{ra}}$, do $L_{\mathrm{rf}}$ e do $L_{\mathrm{rf}}$ acrescido de $3 \mathrm{~dB}$ (ABNT 1995), dia 6

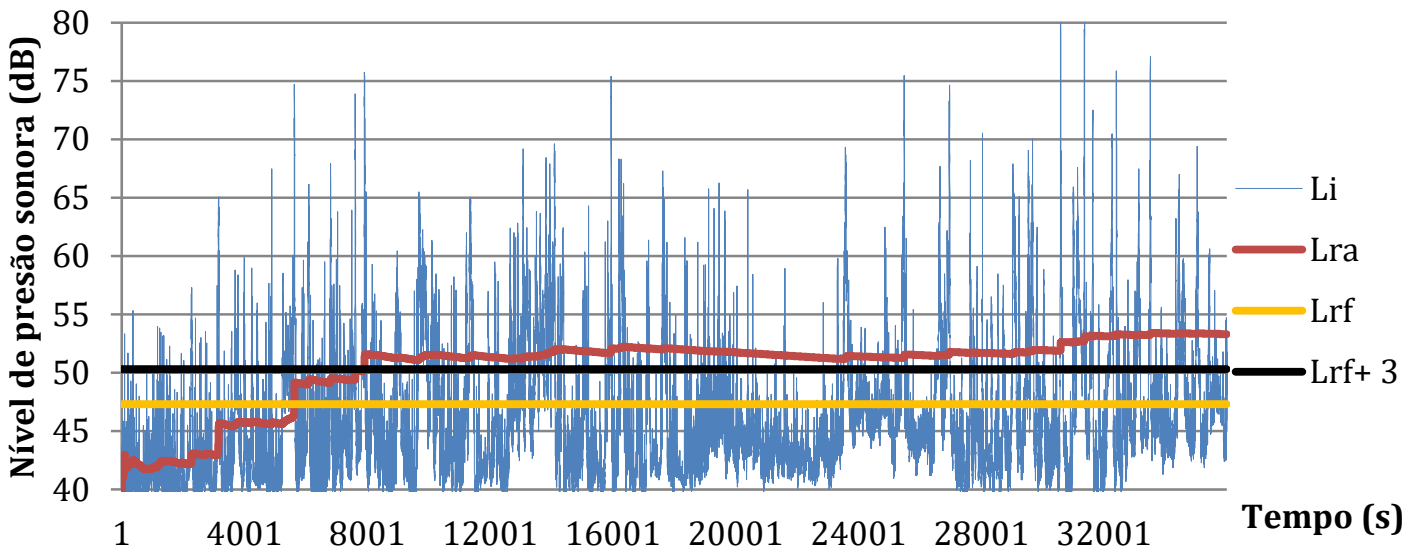

Figura 10 - Histórico do tempo das medidas instantâneas (Li), a evolução do $L_{r a}$, do $L_{r f}$ e do $L_{r f}$ acrescido de 3 dB (ABNT 1995), dia 7

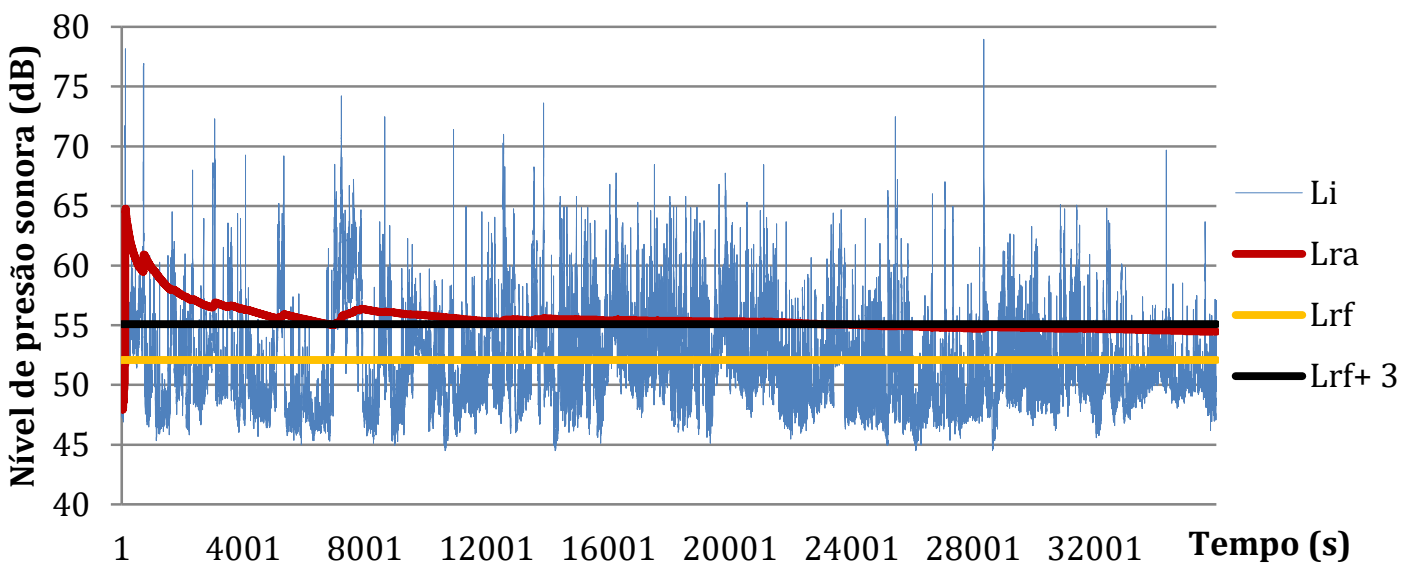

As medidas instantâneas apresentadas nas Figuras 4 a 10 possibilitaram também estimar o tempo médio em que o nível de pressão sonora de uma operação de pouso e decolagem permanece acima de $60 \mathrm{~dB}$, cerca de $30 \mathrm{~s}$. Considerando que o $L_{\mathrm{rf}}$ médio foi de $50,7 \mathrm{~dB}$, o $L_{\max }$ próximo a $80 \mathrm{~dB}$ é totalmente perceptível, principalmente quando ocorrem de 40 a 45 vezes em um período de $10 \mathrm{~h}$, ou de 4 a 5 vezes por hora, ou uma vez a cada $12 \mathrm{~min}$, em valores médios, por um período de cerca de $30 \mathrm{~s}$, o que pode ser o motivo da elevada I\% mesmo em regiões que atendem aos critérios da NBR 13368 (ABNT, 1995), ou da NBR 10151 (ABNT, 2019) ou do zoneamento estabelecido pelo RBAC 161 (AGÊNCIA..., 2003). Conforme mencionado, uma fonte de ruído de $5 \mathrm{~dB}$ inferior à voz humana já pode interferir em uma conversa ou atividade de ensino (BISTAFA; BRADLEY, 2000). Segundo Nunes (2005), o nível de pressão sonora $10 \mathrm{~dB}$ superior ao $L_{\mathrm{r} f}$ gera significativa interferência na comunicação, o que comprova que $30 \mathrm{~dB}$ realmente é uma fonte de incômodo considerável que impacta nas atividades cotidianas da população.

O ruído gerado por fontes urbanas foi o responsável por 396 (56,9\%) ocorrências acima de 60 dB. Em geral, a população tem tolerância maior com o ruído de tráfego, uma das maiores fontes de poluição sonora do meio urbano (FIDEL, 2015). Segundo a WHO (WORLD..., 2011), em pesquisa realizada em 26 países com 34.211 pessoas entrevistadas, o relato de incômodo da população varia de $L_{\mathrm{dn}}$ entre $40 \mathrm{~dB}$ e $80 \mathrm{~dB}$, ou seja, bem diversificado, e indica tendência de maior intolerância para com o ruído aeronáutico em relação ao rodoviário. Observa-se ainda que o ruído no meio urbano também é gerado pela própria população (música, animais domésticos, gritos, conversas), o que seria uma fonte de incômodo, na verdade incorporada ao cotidiano. 
Tabela 9 - Resultados consolidados nos 7 dias de medição para o número de ocorrências e valores de $L_{\max }$ gerados pelo heliporto e por outras fontes de ruído urbano

\begin{tabular}{c|c|c|c|c}
\hline Dia & $\begin{array}{c}\boldsymbol{L}_{\text {max }} \text { gerado pelo } \\
\text { heliporto } \\
\text { Ocorrências }\end{array}$ & $\begin{array}{c}\boldsymbol{L}_{\max } \text { gerado por } \\
\text { fontes urbanas } \\
\text { Ocorrências }\end{array}$ & $\begin{array}{c}\boldsymbol{L}_{\max } \text { gerado pelo } \\
\text { heliporto } \\
(\mathbf{d B})\end{array}$ & $\begin{array}{c}\boldsymbol{L}_{\text {max }} \text { gerado por } \\
\text { fontes urbanas } \\
(\mathbf{d B})\end{array}$ \\
\hline 1 & 28 & 55 & 78,6 & 73,9 \\
2 & 39 & 49 & 75,6 & 68,6 \\
3 & 52 & 40 & 78,7 & 71,7 \\
4 & 49 & 71 & 77,1 & 71,7 \\
5 & 51 & 41 & 79,4 & 71,0 \\
6 & 49 & 46 & 79,5 & 66,2 \\
7 & 32 & 396 & 78,3 & \\
\hline TOTAL & 300 & $56,9 \%$ & & \\
\hline$\%$ & $43,1 \%$ & & & \\
\hline
\end{tabular}

\section{Conclusões}

Este artigo teve o objetivo de avaliar a aplicação das métricas temporais e máximas para se obter o potencial de incômodo devido à poluição sonora gerada por um heliporto no meio urbano. Verificou-se com os resultados obtidos que atender aos critérios estabelecidos pela NBR 13368 (ABNT, 1995) ou NBR 10151 (ABNT, 2019), que utilizam as métricas temporais para avaliação, não garante o conforto acústico da população. A expectativa de resposta de uma população incomodada (I\%) de 30\%, obtida pelas curvas doseresposta utilizadas, indica que existe desconforto. A NBR 13368 (ABNT, 1995), que é específica para a avaliação do ruído gerado por aeronaves, de asas fixas e rotativas, precisa ser atualizada em seus critérios de avaliação.

Os resultados obtidos também apontam que valores de $L_{\max }$ podem ser cerca de $30 \mathrm{~dB}$ superiores ao nível de ruído de fundo $\left(L_{\mathrm{rf}}\right)$, com uma frequência de 12 min e duração de $30 \mathrm{~s}$, em média. Essa ocorrência, repetidas vezes em um dia, pode interferir ou mesmo inviabilizar uma série de atividades cotidianas no momento das operações de pouso e decolagem dos helicópteros, como já mencionado ao longo deste artigo. Dessa forma, é possível relacionar a expectativa de resposta de uma população incomodada ao $L_{\max }$, e não apenas com as métricas temporais utilizadas nas avaliações.

Critérios baseados na diferença entre as métricas temporais e máximas podem ser utilizados a exemplo do que ocorre com o nível de pressão sonora impulsivo, definido na NBR 10151 (ABNT, 2019) como a diferença de $6 \mathrm{~dB}$ entre o $L_{\mathrm{Aeq}}$ (métrica temporal) e o $L_{\max }$ (métrica que considera o nível de pressão sonora máximo). Caso seja identificado o nível de pressão sonora impulsivo, há penalidade de $5 \mathrm{~dB}$, a ser somada na métrica temporal. De maneira semelhante, algum tipo de penalidade poderia ser aplicado no critério de avaliação caso o $L_{\max }$ seja superior ao $L_{\mathrm{rf}}$ acima de um valor ainda a ser estabelecido. Espera-se com isso que os critérios normalizados sejam mais coerentes com a expectativa de resposta de uma população incomodada obtida pelas curvas dose-resposta.

Conclui-se, portanto, que as métricas temporais e os critérios ligados a elas não garantem que a população localizada próximo a aeródromos em geral não seja prejudicada pela poluição sonora e que a incorporação do $L_{\max }$ na avaliação se faz necessária.

\section{Referências}

AGÊNCIA NACIONAL DE AVIAÇÃO CIVIL. Catálogos de aeródromos privados. Disponível em: http://www.anac.gov.br/assuntos/setor-regulado/aerodromos/cadastro-de-aerodromos-civis. Acesso em: 14 fev. 2019.

AGÊNCIA NACIONAL DE AVIAÇÃO CIVIL. Regulamento Brasileiro da Aviação Civil - RBAC 161. Planos de Zoneamento de Ruído de Aeródromos - PZR. Brasília, 2013

ALENCAR, B. S.; OITICICA, M. L. G. Análise do plano de zoneamento de ruído em relação à ocupação do solo no entorno do Aeroporto Internacional Zumbi dos Palmares na Cidade de Maceió-AL. In:

ENCONTRO DA SOCIEDADE BRASILEIRA DE ACÚSTICA, 27., Brasília, 2017. Anais [...] Brasília: Sobrac, 2017. 
ASSOCIAÇÃO BRASILEIRA DE NORMAS TÉCNICAS. NBR 10151: acústica: avaliação do ruído em áreas habitadas, visando o conforto da comunidade: procedimentos. Rio de Janeiro, 2000.

ASSOCIAÇÃO BRASILEIRA DE NORMAS TÉCNICAS. NBR 10151: medição e avaliação de níveis de pressão sonora em áreas habitadas: aplicação de uso geral. Rio de Janeiro, 2019.

ASSOCIAÇÃO BRASILEIRA DE NORMAS TÉCNICAS. NBR 10152: níveis de pressão sonora em ambientes internos a edificações. Rio de Janeiro, 2017.

ASSOCIAÇÃO BRASILEIRA DE NORMAS TÉCNICAS. NBR 13368: ruído gerado por aeronaves: monitoração. Rio de Janeiro, 1995.

BARBOSA, R. A. Influência do ruído aeronáutico na população que reside nas imediações da Base Aérea de Santa Maria. Santa Maria, 2011. Dissertação (Mestrado em Engenharia Civil) - Programa de Pós-Graduação em Engenharia Civil e Ambiental, Universidade Federal de Santa Maria, Santa Maria, 2011.

BERGTRÖN, K. et al. Effects of aircraft noise annoyance and quality of life in German children near Frankfurt Main Airport. In: EURO-NOISE, Maastricht, 2015. Proceedings [...] Maastricht, 2015.

BISTAFA, S. R. Acústica aplicada ao controle do ruído. São Paulo: Edgard Blucher, 2011.

BISTAFA, S. R.; BRADLEY, J. S. Reverberation time and maximum background-noise level for classrooms from a comparative study of speech intelligibility metrics. Journal of the Acoustical Society of America, v. 107, n. 2, p. 861-875, 2000.

BRITO, L. A. P. F. de. A utilização de mapas acústicos como ferramenta de identificação do excesso de ruído em áreas urbanas. Engenharia Sanitária Ambiental, v. 22, n. 6, p. 1095-1107, 2017.

CARVALHO JUNIOR, E. B. Quantificação do incômodo gerado pelo ruído aeronáutico por meio de modelos dose-resposta. Brasília, 2015. Tese (Doutorado em Transportes Urbanos) - Programa de PósGraduação em Transportes, Faculdade de Tecnologia, Universidade de Brasília, Brasília, 2015.

DINATO, A. C. Ruído sonoro no entorno de aeroportos: um estudo de caso no aeroporto de Ribeirão Preto, 2011. Tese (Doutorado em Engenharia Civil) - Universidade de São Paulo, 2011.

EUROPEAN COMISSION. Position paper on dose-response relationships between transportation noise and annoyance - EU's Future Noise Policy, WG2 - Dose/Effect.2002.

EVRARD, A. et al. Does aircraft noise exposure increase the risk of hypertension in the population living near airports in France? Occupational and Environmental Medicine, v. 74, p. 123-129, 2017.

FEDERAL AVIATION ADMINISTRATION. Nonmilitary helicopter urban noise study. Report of the Federal Aviation Administration to the United States Congress.2004.

FEDERAL AVIATION ADMINISTRATION. History of noise. Disponível em: https://www.faa.gov/regulations_policies/policy_guidance/noise/history/. Acesso em: 12 fev. 2019.

FEDERAL INTERAGENCY COMMITTEE ON NOISE. Federal Government's Method of Assessing Noise Impacts. Washington DC, 1992.

FIDEL, S.A review of US Aircraft Noise Regulatory Policy. Acoustical Society of America - Acoustic Today, v. 11, n. 4, p. 26-34, 2015.

LAWTON, R. N.; FUJIWARA, D. Living with aircraft noise: airport proximity, aviation noise and subjective wellbeing in England. Transportation Research Part D: Transport and Environment, v. 42, p. 104-118, 2016.

LEFÈVRE, E. A. S. M.; LAMBERT, P. C. H.; LAUMON, B. Does exposure to aircraft noise increase the risk of hypertension near the French airports? Occupational and Environmental Medicine, v. 74, p. 123 129, 2017.

MARQUES, J. R. Os danos causados ao meio ambiente por poluição sonora, eletromagnética, visual e luminosa: reparação, sanções penais e Administrativas. Revista Jurídica do Ministério Público do Estado de Minas Gerais, Belo Horizonte, v. 14, n. 25, p. 217-296, jul./dez. 2015.

MURPHY, E. Environmental noise pollution: noise mapping, public health, and policy. Dublin: Elsevier, 2014. 
NUNES, M. F. O. Avaliação da percepção do ruído aeronáutico em escolas. Porto Alegre, 2005. Tese (Doutorado em Engenharia Civil) - Programa de Pós Graduação em Engenharia Civil, Universidade Federal do Rio Grande do Sul, Porto Alegre, 2005.

PEREIRA, L. F. M. Análise de heliportos civis para operação do EH-101. Pedrouços, 2018. Dissertação (Mestrado em Estudos Militares) - Departamento de Estudos Pós-Graduados, Instituto Universitário Militar, Pedrouços, 2018.

ROSOLEM, J.; HENKES, J. A. Avaliação do impacto ambiental causado pelos ruídos sonoros provenientes do Aeroporto de Guarulhos: impactos negativos ao homem e ao meio ambiente. Revista Gestão e Sustentabilidade Ambiental, Florianópolis, v. 6, n. 3, p. 634-650, out./dez. 2017.

SANTOS, G. B. Análise espacial dos fatores determinantes no cálculo da área impactada pelo ruído aeronáutico. São José dos Campos, 2015. Tese (Doutorado em Infraestrutura Aeronáutica) - Instituto de Engenharia Aeronáutica, São José dos Campos, 2015.

SCHRECKENBERG, D. et al. The relationship between aircraft sound levels, noise annoyance and mental well-being: an analysis of moderated mediation. In: ICBEN CONGRESS ON NOISE AS A PUBLIC HEALTH PROBLEM, 12., Zurich, 2017. Proceedings [...] Zurich, 2017.

SCHULTZ, T. J. Synthesis of social surveys on noise annoyance. Journal of the Acoustical Society of America, v. 64, n. 2, p. 377-405, 1978.

SHOEGIMA, T. F. Poluição sonora urbana: estudo de caso da Subprefeitura de Pinheiros. São Paulo, 2011. Dissertação (Mestrado em Geografia Física) - Faculdade de Filosofia, Letras e Ciências Humanas, Programa de Pós-Graduação em Geografia Física, Universidade de São Paulo, São Paulo, 2011.

SILVA, B. G. C da. Development of methodology of evaluation and communication of aircraft noise impacts around airports. São José dos Campos, 2016. Tese (Doutorado em Infraestrutura Aeronáutica) Instituto de Engenharia Aeronáutica, São José dos Campos, 2016.

VASCIK, P. D.; HANSMAN, R. J. Scaling constraints for urban air mobility operations: air traffic control, ground infrastructure, and noise. In: AIAA AVIATION TECHNOLOGY, INTEGRATION, AND OPERATIONS CONFERENCE, 18., Atlanta, 2018. Proceedings [...] Atlanta, 2018.

WORLD HEALTH ORGANIZATION. Burden of disease from environmental noise: quantification of healthy life years lost in Europe. Denmark, 2011.

Luiz Antonio Perrone Ferreira de Brito

Programa de Pós Graduação e Gestão e Desenvolvimento Regional | Universidade de Taubaté | Rua Quatro de Março, 432 | Taubaté - SP Brasil | CEP 12020-270 | Tel.: (12) 3621-8839 | E-mail: labrito@bighost.com.br

Rafael Felipe Guatura

Programa de Pós Graduação e Gestão e Desenvolvimento Regional | Universidade de Taubaté | E-mail: eng.rguatura@gmail.com

João Pedro Reis da Silva

Departamento de Arquitetura Universidade de Taubaté | E-mail: joao_21pedro@hotmail.com

\section{Ambiente Construído}

Revista da Associação Nacional de Tecnologia do Ambiente Construído

Av. Osvaldo Aranha, $99-3^{\circ}$ andar, Centro

Porto Alegre - RS - Brasil CEP $90035-190$

Telefone: +55 (51) 3308-4084

Fax: +55 (51) 3308-4054

www.seer.ufrgs.br/ambienteconstruido

E-mail: ambienteconstruido@ufrgs.br

This is an open-access article distributed under the terms of the Creative Commons Attribution License. 